\title{
Maintenance therapies for non-small cell lung cancer
}

\author{
Normand Blais* and Elie Kassouf \\ Medical Oncology, Centre Hospitalier de l'Université de Montréal, Montreal, QC, Canada
}

\section{Edited by:}

Vera Hirsh, McGill University Health

Centre, Canada

Reviewed by:

Sacha I. Rothschild, University Hospital Basel, Switzerland

Janaki Deepak, University of

Maryland School of Medicine, USA

*Correspondence:

Normand Blais, Medical Oncology,

Centre Hospitalier de I'Université de

Montréal, 1560, Sherbrooke Est,

Montreal, Quebec, Canada

e-mail:n.blais@umontreal.ca
Treatment of lung cancer had evolved during the last decade with the introduction of new chemotherapeutic regimens and targeted therapies. However, the maximum benefit reached after first-line therapy is limited by the cumulative toxicity of platinum drugs and the subsequent deterioration in performance status in a high percentage of patients who end up receiving not more than one line of treatment. Maintenance therapy had been introduced and evaluated in many large randomized trials showing a delay in tumor progression and an improvement in overall survival. This effective strategy should be taken into account when discussing the initial treatment plan and tailored according to the preferences of both patients and physicians.

Keywords: maintenance, pemetrexed, erlotinib, gefitinib, bevacizumab, lung cancer, docetaxel

\section{INTRODUCTION}

Standard first-line treatment for patients who are negative for the EGFR mutation and ALK rearrangement consists of platinumbased chemotherapy (1). The optimal number of cycles had been determined after Park et al. compared four to six cycles of treatment showing non-inferiority in terms of overall survival and thus, making four cycles a currently accepted standard (2).

During the past decade, research from different work groups has focused on finding alternative strategies to improve tumor response and extend survival.

The limited benefit from extending platinum-based chemotherapy beyond four cycles as well as the cumulative toxicities of these regimens leading to worsening of the quality of life (QoL) (2-4) had led to the re-emergence of a relatively new concept based on maintaining the response in patients who attain tumor control during first-line induction treatment.

Therefore, cytotoxic agents and molecularly targeted agents have been extensively evaluated in this setting and two practical applications of maintenance therapy have evolved: continuation maintenance and switch maintenance.

Maintenance therapy has potential advantages and inconveniences. Although many large randomized studies have shown that maintenance therapy is associated with a delay in tumor progression and an improvement in overall survival, prolonging therapy in a palliative intent also significantly increases the burden of medical interventions to a patient, prohibits the patient from having an often desired treatment holiday and may also increase treatment related toxicities, which may have a detrimental impact on QoL.

This mini review will expose the issues related to maintenance therapy and discuss a personalized approach to the implementation of such strategies in clinical practice.

\section{CONTINUATION MAINTENANCE}

Continuation maintenance refers to the continuation of one or more non-platinum agents, initially used during induction, until progression. This approach allows the discontinuation of platinum compounds known to cause cumulative toxicity that often becomes clinically meaningful after four to six doses of these drugs. It also has the advantage of continuing an agent for which tolerance has been defined in the induction phase of the treatment.

In 2006, Sandler et al. published the results of the ECOG 4599 trial, which showed that continuing bevacizumab (Bev) beyond six cycles of paclitaxel (Pac)/carboplatin (Carbo)/Bev added 2 months improvement in OS compared to six cycles of Carbo/Pac alone (HR, 0.79, $p=0.003$ ) (5). Similarly, in 2009, Pirker et al. demonstrated in the FLEX study, that maintenance with cetuximab after chemotherapy with cisplatin/vinorelbine/cetuximab significantly improved OS (11.3 vs. 10.1 months; HR, $0.87, p=0.044)$ compared to cisplatin/vinorelbine alone (6). It is not clear whether the benefit of bevacizumab or cetuximab in these studies is from their integration of the induction phase, the maintenance phase or both. Nonetheless, the design of these trials revived the concept of adapting maintenance as an appropriate clinical strategy.

Gemcitabine was evaluated in three randomized trials. CECOG (7) and IFCT-GFPC 0502 (8) met their primary endpoint showing longer PFS but with no significantly improved OS. These trials show interesting trends toward an increase in overall survival although this interpretation is limited by the small sample size of these trials. In a third trial, Belani et al. failed to demonstrate any advantage of maintenance gemcitabine on PFS and OS. These negative results have been attributed to the fact that most patients (64\%) had a poor performance status (PS 2) at randomization in contrast to most other maintenance trials where most of the patients were PS 0 or 1 (9).

More recently, continuation maintenance with pemetrexed (pem) has been evaluated in two large randomized studies. In the PARAMOUNT trial, continuation pem after four cycles of induction with cisplatin/pem demonstrated significant improvement in PFS (4.1 vs. 2.8 months; HR, $0.62 ; p<0.0001)$ and OS (16.9 vs. 14 months; HR, 0.78; $p=0.019$ ) (10). In 2009, Patel et al. combined pem and bev as continuous maintenance in a phase II study. Their strategy was safe and resulted in a promising 14.1 months median OS (11). Maintenance with pem and bev was 
thereafter tested in three major phase III studies. In AVAPERL, there was a 3.6 months improvement in PFS in the Pem/Bev arm compared to the Bev arm, and OS benefit was similar as that reported in PARAMOUNT although the sample size was too small to demonstrate statistical significance (19.8 vs. 15.9 months, HR, $0.88, p=0.32)(12)$.

Although not a maintenance trial per se, PointBreak compared the ECOG 4599 regimen Pac/Carbo/Bev followed by Bev to Pem/Carbo/Bev followed by maintenance with Pem/Bev. Despite the fact that this study did not show significant improvement in OS (median OS, 12.6 vs. 13.4 months; $p=0.949$ ) and a slight advantage in PFS favoring the pemetrexed containing regimen (median PFS, 6.0 vs. 5.6 months; HR, 0.83 ; $p=0.012$ ), the study results highlighted different toxicity profiles between the paclitaxel and pemetrexed containing regimens that may lead to a better treatment selection (13). Fatigue and thrombocytopenia were more frequent in the pem arm and neutropenia, neuropathy, and alopecia more frequent in the paclitaxel arm.

The ongoing ECOG 5508 study may better define the optimal choice of maintenance agent after pac/carbo/bev as it randomizes patients for maintenance after induction into three arms: Bev alone, Pem alone, or combined Pem-Bev (Table 1).

\section{SWITCH MAINTENANCE}

Switch maintenance is the introduction of an additional, potentially non-cross-resistant agent, immediately after completion of first-line chemotherapy in patients who achieved an objective response or a stable disease. This strategy focuses on the early integration of drugs that have been shown to be useful in the second line setting and in this regard can be seen as "early second line" therapy. This exposes the patient to new toxicity that needs to be addressed before choosing such a strategy.

The first pivotal trial to report benefit with a cytotoxic agent using this strategy was presented by Fidias et al. who randomized 309 patients with advanced NSCLC who did not progress after front-line treatment with four cycles of carbo/gemcitabine to receive immediate docetaxel maintenance therapy vs. delayed docetaxel at disease progression. The study showed a significant 3 months improvement in PFS and a non-statistically significant 2.5 months increase in OS in favor of the "immediate" docetaxel arm, with no increase in toxicity or decrease in QOL. Even though the patients in the delayed arm were carefully assessed and followed and that docetaxel was available to all of these patients, $37.2 \%$ of the patients in this arm did not receive docetaxel due to a rapid disease progression or a rapid PS decline (14).

JMEN is a phase III trial that evaluated maintenance pem vs. BSC in 633 non-progressive stage IIIB/IV patients after non-pem containing platinum-doublet chemotherapy. The pemetrexed arm showed significantly improved PFS (4.3 vs. 2.6 months; HR, 0.5, $p<0.0001)$ and OS (13.4 vs. 10.6 months; HR, $0.79 ; p=0.012)$. Subgroup analysis based on histology showed that the improvement in PFS ( 4.5 vs. 2.6 months; HR, $0.44 ; p=0.0001$ ) and OS (15.5 vs. 10.3 months; HR, $0.70 ; p=0.002$ ) was restricted to patients with tumors having a non-squamous histology $(72.5 \%$ of the population) (15). In this trial, there was a limited access to pem in the BSC arm, as only $18 \%$ of patients were treated with this drug, thus creating a subsequent imbalance in interpreting the benefit of pem as a highly active drug in this particular population.

After showing an OS benefit in second and third line setting for advanced NSCLC (16), erlotinib was evaluated as a switch

Table 1 | Key studies addressing continuous maintenance.

\begin{tabular}{|c|c|c|c|c|c|c|c|c|}
\hline Reference (study name) & $N$ (pts) & Maintenance arms & PFS (mo) & HR & $p$ Value & OS (mo) & HR & $p$ Value \\
\hline Sandler et al. (5) & 850 & Bevacizumab & 6.2 & 0.66 & $<0.001$ & 12.3 & 0.79 & 0.003 \\
\hline (ECOG 4599) & & Observation & 4.5 & & & 10.3 & & \\
\hline Pirker et al. (6) & 850 & Cetuximab & 4.8 & 0.943 & 0.39 & 11.3 & 0.871 & 0.044 \\
\hline (FLEX) & & Placebo & 4.8 & & & 10.1 & & \\
\hline Brodowicz et al. (7) & 206 & Gemcitabine & 3.6 & & $<0.001$ & 13 & & 0.195 \\
\hline (CECOG) & & BSC & 2.0 & & & 11 & & \\
\hline Perol et al. (8) & 464 & Gemacitabine & 3.8 & 0.56 & $<0.001$ & 12.1 & 0.89 & 0.3867 \\
\hline (IFCT-GFPC 0502) & & Observation & 1.9 & & & 10.8 & & \\
\hline \multirow[t]{2}{*}{ Belani et al. (9) } & 255 & Gemacitabine & 3.9 & & 0.58 & 8.0 & 0.97 & 0.84 \\
\hline & & Observation & 3.8 & & & 9.3 & & \\
\hline Paz-Ares et al. (10) & 539 & Pemetrexed & 4.1 & 0.62 & $<0.001$ & 13.9 & 0.78 & 0.0195 \\
\hline (PARAMOUNT) & & BSC & 2.8 & & & 11.0 & & \\
\hline Barlesi et al. (12) & 253 & Pem/Bev & 10.2 & 0.5 & $<0.001$ & 19.8 & 0.88 & 0.32 \\
\hline (AVAPERL) & & Bev & 6.6 & & & 15.9 & & \\
\hline Patel et al. (13) & 590 & Pem/Bev & 6.0 & 0.83 & 0.012 & 12.6 & 1 & 0.949 \\
\hline (Point break) & & Bev & 5.6 & & & 13.4 & & \\
\hline
\end{tabular}

PFS = progression-free survival; $O S=$ overall survival; $B S C=$ best supportive care; $H R=$ hazard ratio; Bev= bevacizumab; Pem = pemetrexed 
maintenance therapy in SATURN, a large phase III trial that randomized 889 patients who did not progress after four cycles of a platinum doublet, to erlotinib or placebo. There was a modest but statistically significant improvement in PFS (3 vs. 2.8 months; HR $0.71, p<0.0001$ ) and OS (12 vs. 11 months; HR, $0.81, p=0.0088$ ). Subgroup analyses showed larger treatment benefit in terms of OS in patients with stable disease (HR, 0.72) after induction than in responders (HR, 0.94). Progression-free survival was significantly higher in patients with EGFR activating mutations (HR, 0.23) than in patients EGFR WT (HR, 0.78) but a survival difference could not be demonstrated in these subgroups (17). In a similar fashion to the JMEN trial, erlotinib was not widely available to patients in the placebo arm and only $21 \%$ of these patients were actually treated with erlotinib. Similar results were seen in the Erlotinib maintenance arm of the smaller IFCT-GFPC 0502 study mentioned earlier, with a 1 month improvement in PFS but no statistically significant change in OS (8). Following these positive results, Johnson et al. studied the combination of bev and erlotinib in maintenance. In the ATLAS trial, 1160 patients received firstline platinum-based chemotherapy with bev, 768 patients had an objective response or $\mathrm{SD}$, and were randomized to receive bev with erlotinib vs. bev alone. This trial showed 1 month improvement in PFS (4.8 vs. 3.7 months, HR 0.72, $p=0.0012$ ) for patients in the combination maintenance arm but a non-statistically significant improvement in OS (14.4 vs. 13.3 months; HR, 0.92; $p=0.5341)(18)$.

The EORTC Lung Cancer Group and the Italian Lung Cancer Project evaluated maintenance with gefitinib vs. placebo in 173 patients who did not progress after four cycles of platinum-based chemotherapy. PFS was better in the treatment arm (4.1 vs. 2.9 months; HR, $0.61 ; p=0.0015)$ but with no statistically significant improvement in OS (10.9 vs. 9.4 months; HR, $0.83 ; p=0.2$ ) (19). The INFORM trial, an Asian phase III trial, tested gefitinib in a similar setting in 296 patients (79 EGFR-mut). PFS was significantly higher in the gefitinib arm (4.8 vs. 2.6 months; HR, $0.42 ; p<0.0001)$ with more benefit EGFR-mut subgroup (HR, $0.17)$ compared to EGFR WT (HR, 0.87). An OS benefit was not shown (18.7 vs. 16.9 months; HR, $0.84 ; p=0.2608$ ) (20). Finally, the West Japan Thoracic Oncology Group 0203 phase III trial compared prolonged chemotherapy with 6 cycles of a platinum doublet to a short course of 3 cycles followed by gefitinib maintenance in 604 patients. PFS was statistically better favoring gefitinib maintenance ( 4.6 vs. 4.3 months; HR, 0.68 ; $p<0.001$ ) but no significant difference in OS was found (13.7 vs. 12.9 months; HR, $0.86 ; p=0.11)(21)$ (Table 2).

\section{DISCUSSION}

Maintenance therapy, whether in switch or continuation approach, has proved to be beneficial in patients with advanced NSCLC who have received up to four cycles of a platinum-containing regimen. Despite much debate regarding the results of the different studies and the reserved improvement in survival, Pemetrexed and Erlotinib are already approved and used for maintenance in many countries.

\section{ARGUMENTS AGAINST MAINTENANCE}

In dealing with a population of patients with a non-curable disease, improvements in overall survival and QoL remain the primary

Table 2 | Key studies evaluating switch maintenance.

\begin{tabular}{|c|c|c|c|c|c|c|c|c|}
\hline Reference (study name) & $N($ pts) & Maintenance arms & PFS (mo) & HR & $p$ Value & OS (mo) & HR & $p$ Value \\
\hline \multirow[t]{3}{*}{ Fidias et al. (14) } & 309 & Immediate & 5.7 & & 0.0001 & 12.3 & & 0.0853 \\
\hline & & Docetaxel & & & & & & \\
\hline & & Docetaxel & & & & & & \\
\hline Ciuleanu et al. (15) & 663 & Pemetrexed & 4.3 & 0.5 & $<0.0001$ & 13.4 & 0.79 & 0.012 \\
\hline Cappuzzo et al. (17) & 889 & Erlotinib & 3.0 & 0.71 & $<0.0001$ & 12.0 & 0.81 & 0.0088 \\
\hline (SATURN) & & Placebo & 2.8 & & & 11.0 & & \\
\hline Perol et al. (8) & 310 & Erlotinib & 2.9 & 0.69 & 0.003 & 11.4 & 0.87 & 0.3043 \\
\hline (IFCT-GFPC 0502) & & Observation & 1.9 & & & 10.8 & & \\
\hline (EORTC08021-LCP01/03) & & Placebo & 2.9 & & & 9.4 & & \\
\hline Zhang et al. (20) & 296 & Gefitinib & 4.8 & 0.42 & $<0.0001$ & 18.7 & 0.84 & 0.26 \\
\hline (INFORM) & & Placebo & 2.6 & & & 16.9 & & \\
\hline Takeda et al. (21) & 604 & Gefitinib & 4.6 & 0.68 & $<0.001$ & 13.7 & 0.86 & 0.11 \\
\hline (WJTOG 0203) & & Observation & 4.3 & & & 12.9 & & \\
\hline
\end{tabular}

$P F S=$ progression-free survival; $O S=$ overall survival; $B S C=$ best supportive care; $H R=$ hazard ratio; $B e v=$ bevacizumab; $P$ em $=$ pemetrexed 
objective. Symptomatic patients who begin induction therapy with a platinum doublet for lung cancer are often looking forward to a symptom free and drug free holiday. In this regard, many patients who obtain a meaningful symptomatic response to induction are not enthusiastic about adding on more therapy, especially if maintenance therapy is discussed after induction. Compared to continuation maintenance, switch maintenance has the added inconvenience of exposing the patient to new toxicities not encountered during induction. The interpretation of many trials is also bound with controversy. In particular, the absence of broad availability of pemetrexed in the JMEN trial or erlotinib in SATURN limits the interpretation of any small OS gain observed in these studies as these drugs are now widely available in many countries, particularly in the second line setting. The question now becomes as to whether these agents are better given before radiological or clinical progression ("early second line") or at the time of clear progression.

\section{ARGUMENTS FOR MAINTENANCE}

The current data appear even more compelling for continuation maintenance, especially for non-squamous and EGFR-mut patients. The PARAMOUNT trial has shown a PFS and an OS benefit for continuation pem in responding and stable disease patients. As used in this trial, limiting therapy to four cycles of cisplatin therapy decreases platin-related toxicities and the absence of new drug exposure limits the risk of new unexpected toxicities. Considering a median time to progression of 6-12 weeks in many observation arms of maintenance strategy trials, delaying progression is a clinically meaningful endpoint to many patients.

The case for switch maintenance is more debatable. For reasons described above, the apparent OS benefit for switching to erlotinib or pemetrexed may be associated to study design not relevant to current practice. Nonetheless, progression in the placebo arms is often very rapid as reported in trials where radiological and clinical follow up is frequent. Leaving patients without treatment can thus expose them to rapid and early progression, often leading to a decline in performance status and inability to receive further therapy. As the biggest benefit in the JMEN and SATURN trial appears to be in patients obtaining no more than SD to induction therapy, it may be hypothesized that these patients may obtain more benefit from an earlier initiation of second line therapy. As such, it seems reasonable to consider switch therapy to patients that did not obtain palliative benefit from induction therapy in an attempt to better alleviate symptoms and prevent symptomatic progression.

Some patients are identified as having an actionable mutation during induction chemotherapy. The ideal timing of the beginning of targeted therapy in this particular situation is still a matter of debate. It seems reasonable to pursue induction chemotherapy in these patients, particularly if they are responding and tolerating treatment well. On the other hand, switching to a specific targeted agent is appropriate if induction is poorly tolerated or if symptoms are poorly controlled. Targeted agents, for instance EGFR and ALK inhibitors, are associated with rapid improvement in symptoms in patients harboring sensitive mutations to these agents.

\section{CONCLUSION}

Maintenance therapy has shown effectiveness in delaying progression in many studies as well as prolonging overall survival in some settings. Appropriate clinical decisions involve early discussions of these options with potentially eligible patients. Factors that may impact in the final decision to initiate maintenance include tumor histology, clinical and radiological response to induction, tumor mutations, and most importantly patient choice. Further improvements in treatment and patient selection will most likely arise with the improved refinement of the molecular diagnosis of lung cancers.

\section{REFERENCES}

1. National Comprehensive Cancer Network. Non-small cell lung cancer (version 3.2014). (2014). Available: http://www.nccn.org/professionals/physician_gls/

2. Park JO, Kim SW, Ahn JS, Suh C, Lee JS, Jang JS, et al. Phase III trial of two versus four additional cycles in patients who are non progressive after two cycles of platinum-based chemotherapy in non small-cell lung cancer. J Clin Oncol (2007) 25(33):5233-9. doi:10.1200/JCO.2007.10.8134

3. Soon YY, Stockler MR, Askie LM, Boyer MJ. Duration of chemotherapy for advanced non-small-cell lung cancer: a systematic review and meta-analysis of randomized trials. J Clin Oncol (2009) 27:3277-83. doi:10.1200/JCO.2008.19. 4522

4. Socinski MA, Schell MJ, Peterman A, Bakri K, Yates S, Gitten R, et al. Phase III trial comparing a defined duration of therapy versus continuous therapy followed by second-line therapy in advanced IIIB/IV non-small-cell lung cancer. J Clin Oncol (2002) 20:1335-43. doi:10.1200/JCO.20.5.1335

5. Sandler A, Gray R, Perry MC, Brahmer J, Schiller JH, Dowlati A, et al. Paclitaxelcarboplatin alone or with bevacizumab for non-small-cell lung cancer. N Engl J Med (2006) 355:2542-50. doi:10.1056/NEJMoa061884

6. Pirker R, Pereira JR, Szczesna A, von Pawel J, Krzakowski M, Ramlau R, et al. Cetuximab plus chemotherapy in patients with advanced non-small-cell lung cancer (FLEX): an open-label randomised phase III trial. Lancet (2009) 373:1525-31. doi:10.1016/S0140-6736(09)60569-9

7. Brodowicz T, Krzakowski M, Zwitter M, Tzekova V, Ramlau R, Ghilezan N, et al. Cisplatin and gemcitabine first-line chemotherapy followed by maintenance gemcitabine or best supportive care in advanced non-small cell lung cancer: a phase III trial. Lung Cancer (2006) 52:155-63. doi:10.1016/j.lungcan. 2006.01.006

8. Perol M, Chouaid C, Perol D, Barlesi F, Gervais R, Westeel V, et al. Randomized, phase III study of gemcitabine or erlotinib maintenance therapy versus observation, with predefined second-line treatment, after cisplatin gemcitabine induction chemotherapy in advanced non-small-cell lung cancer. J Clin Oncol (2012) 30:3516-24. doi:10.1200/JCO.2011.39.9782

9. Belani CP, Waterhouse DM, Ghazal H, Ramalingam SS, Bordoni R, Greenberg R, et al. Phase III study of maintenance gemcitabine (G) and best supportive care (BSC) versus BSC, following standard combination therapy with gemcitabine-carboplatin (G-Cb) for patients with advanced non-small cell lung cancer (NSCLC). J Clin Oncol (2010) 28(15 Suppl):abstr 7506.

10. Paz-Ares L, de Marinis F, Dediu M, Thomas M, Pujol JL, Bidoli P, et al. Maintenance therapy with pemetrexed plus best supportive care versus placebo plus best supportive care after induction therapy with pemetrexed plus cisplatin for advanced non-squamous non-small-cell lung cancer (PARAMOUNT): a double-blind, phase 3, randomized controlled trial. Lancet Oncol (2012) 13:247-55. doi:10.1016/S1470-2045(12)70063-3

11. Patel JD, Hensing TA, Rademaker A, Hart EM, Blum MG, Milton DT, et al. Phase II study of pemetrexed and carboplatin plus bevacizumab with maintenance pemetrexed and bevacizumab as first-line therapy for nonsquamous non-smallcell lung cancer. J Clin Oncol (2009) 27:3284-9. doi:10.1200/JCO.2008.20.8181

12. Barlesi F, De Castro J, Dvornichenko V, Kim J, Pazzola A, Rittmeyer A, et al. AVAPERL (MO22089): final efficacy outcomes for patients with advanced nonsquamous non small cell lung cancer randomized to continuation maintenance with bevacizumab (Bev) or Bev + pemetrexed (Pem) after first-line Bevcisplatin-Pem treatment. Eur J Cancer (Oxford) (2013) 47:16. doi:10.1016/j.cllc. 2011.05.002 
13. Patel J, Socinski M, Garon E, Reynolds C, Spigel D, Olsen M, et al. PointBreak: a randomized phase III study of pemetrexed plus carboplatin and bevacizumab followed by maintenance pemetrexed and bevacizumab versus paclitaxel plus carboplatin and bevacizumab followed by maintenance bevacizumab in patients with stage IIIB or IV nonsquamous non-small-cell. Lung Cancer JCO (2013) 2012:47.9626. doi:10.1200/JCO.2012.47.9626

14. Fidias PM, Dakhil SR, Lyss AP, Loesch DM, Waterhouse DM, Bromund JL, et al. Phase III study of immediate compared with delayed docetaxel after front-line therapy with gemcitabine plus carboplatin in advanced non-small-cell lung cancer. J Clin Oncol (2009) 27:591-8. doi:10.1200/JCO.2008.17.1405

15. Ciuleanu T, Brodowicz T, Zielinski C, Kim JH, Krzakowski M, Laack E, et al. Maintenance pemetrexed plus best supportive care versus placebo plus best supportive care for non-small-cell lung cancer: a randomized, double-blind, phase 3 study. Lancet (2009) 374:1432-40. doi:10.1016/S0140-6736(09)61497-5

16. Sheperd F, Pereira JR, Ciuleanu T, Tan EH, Hirsh V, Thongprasert S, et al. Erlotinib in previously treated non-small-cell lung cancer. N Engl J Med (2005) 353:123-32. doi:10.1056/NEJMoa050753

17. Cappuzzo F, Ciuleanu T, Stelmakh L, Cicenas S, Szczesna A, Juhasz E, et al. Erlotinib as maintenance treatment in advanced non-small-cell lung cancer: a multicentre, randomized, placebo-controlled phase 3 study. Lancet Oncol (2010) 11:521-9. doi:10.1016/S1470-2045(10)70112-1

18. Johnson B, Kabbinavar F, Fehrenbacher L, Hainsworth J, Kasubhai S, Kressel B, et al. ATLAS: randomized, double-blind, placebo-controlled, phase IIIB trial comparing bevacizumab therapy with or without erlotinib, after completion of chemotherapy, with bevacizumab for first-line treatment of advanced nonsmall-cell lung cancer. Lung Cancer JCO (2013) 2012:47.3983. doi:10.1200/JCO. 2012.47.3983

19. Gaafar RM, Surmont VF, Scagliotti GV, Van Klaveren RJ, Papamichael D, Welch JJ, et al. A double-blind, randomized, placebo-controlled phase III intergroup study of gefitinib in patients with advanced NSCLC, non-progressing after first line platinum-based chemotherapy (EORTC 08021/ILCP 01/03). Euro J Cancer (2011) 47(15):2331-40. doi:10.1016/j.ejca.2011.06.045

20. Zhang L, Shenglin M, Song X, Han B, Cheng Y, Huang C, et al. Efficacy, tolerability, and biomarker analyses from a phase III, randomized, placebo-controlled, parallel group study of gefitinib as maintenance therapy in patients with locally advanced or metastatic NSCLC. J Clin Oncol (2011) 29:abstr LBA7511.

21. Takeda K, Hida T, Sato T, Ando M, Seto T, Satouchi M, et al. Randomized phase III trial of platinum-doublet chemotherapy followed by gefitinib compared with continued platinum-doublet chemotherapy in Japanese patients with advanced non-small-cell lung cancer: WJTOG0203. J Clin Oncol (2010) 28:753-60. doi:10.1200/JCO.2009.23.3445

Conflict of Interest Statement: The Guest Associate Editor Vera Hirsh declares that, despite having collaborated with author Normand Blais, the review process was handled objectively and no conflict of interest exists. The authors declare that the research was conducted in the absence of any commercial or financial relationships that could be construed as a potential conflict of interest.

Received: 12 May 2014; accepted: 25 July 2014; published online: 19 August 2014. Citation: Blais N and Kassouf E (2014) Maintenance therapies for non-small cell lung cancer. Front. Oncol. 4:213. doi: 10.3389/fonc.2014.00213

This article was submitted to Thoracic Oncology, a section of the journal Frontiers in Oncology.

Copyright (c) 2014 Blais and Kassouf. This is an open-access article distributed under the terms of the Creative Commons Attribution License (CC BY). The use, distribution or reproduction in other forums is permitted, provided the original author (s) or licensor are credited and that the original publication in this journal is cited, in accordance with accepted academic practice. No use, distribution or reproduction is permitted which does not comply with these terms. 